\title{
Mindful Melody: Feasibility of the use of music to reduce agitation on an acute inpatient psychiatric floor.
}

\author{
L. Leontieva, T. Scudamore, A. Liem, M. Wiener, S. Ekure, C. Botash, \& D. Empey \\ State University of New York, Upstate Medical University
}

Syracuse, NY USA

\section{Background}

- Music has enormous ability to influence human emotions. It could mirror them, and consequently change the mood of their listeners.

- Studies to date suggest that music can be used as an alternative therapy for treating psychiatric depression, autism, schizophrenia, dementia and their symptoms such as agitation, insomnia, and anxiety.

- Definition of agitation is: an "excessive verbal and/or motor behavior" that can be loud, disruptive, hostile, sarcastic, threatening, hyperactive, and/or combative"5

- Thus, agitation is an attempt to communicate an unmet psychosocial needs.

- On acute psychiatric floors agitation is typically managed with verbal de-escalation first, followed by as-needed psychotropic medications (PRNs) administration: oral first, if patient refuses and behavior escalates to being dangerous to self/others - intramuscular medication administration.

- Studies show casual use of PRNs for agitation may lead to unnecessary exposures at increasing doses, especially in patients with personality disorders. 2,3

- Music therapy has been previously ranked as the independent therapeutic nursing intervention most often used to decrease agitation, aggression, and depression as well as enhance sleep. ${ }^{4}$

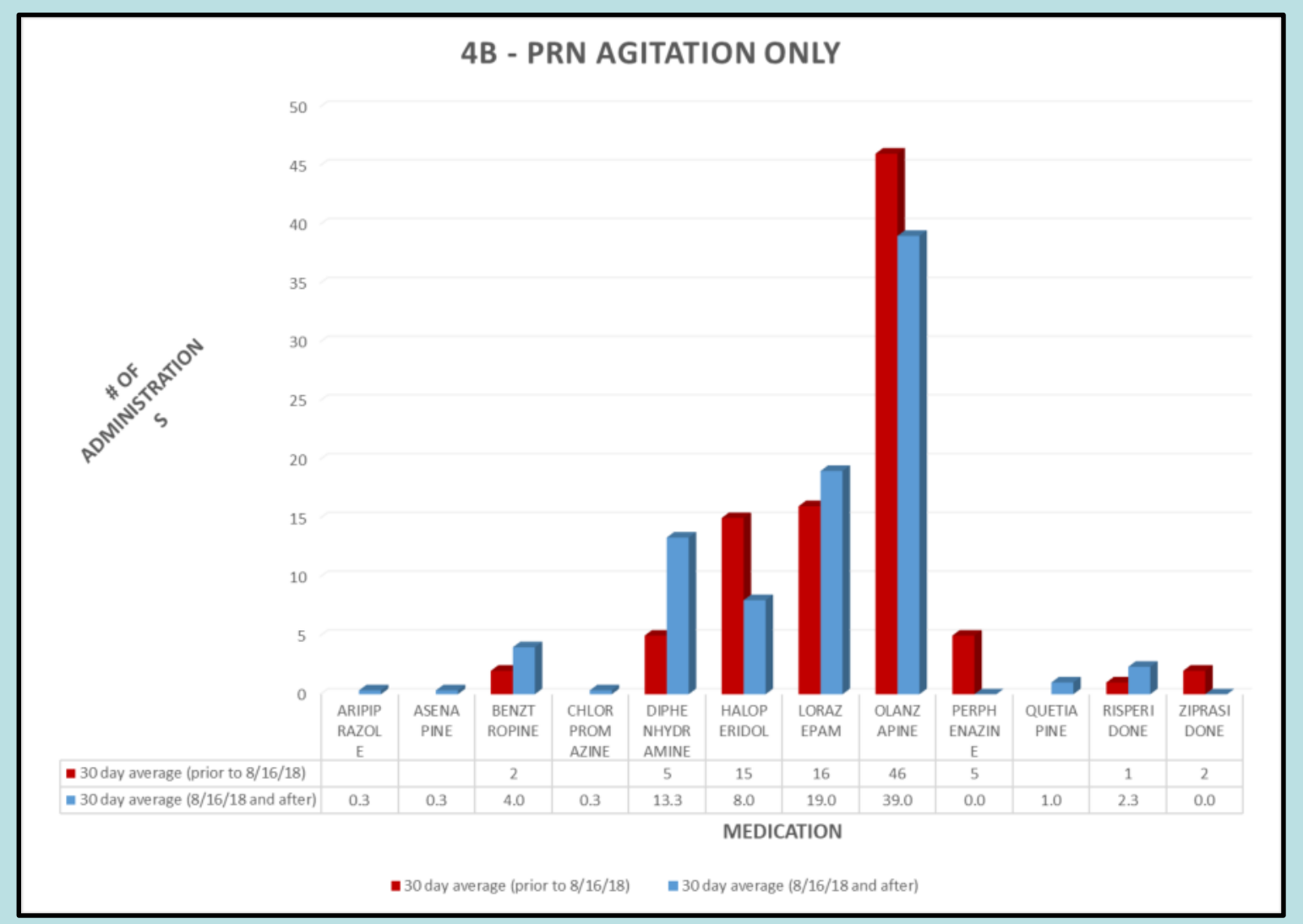

- $56 \%$ of surveyed nursing staff felt PRN music helped patients calm down. Those disagreeing indicated increased intrusive behavior at the nursing station among some patients utilizing the music. These intrusive behaviors decreased as patients learned appropriate boundaries.

- $96 \%$ of surveyed patients responded with positive feedback regarding their experience using PRN music for agitation reduction. Some patients reported being able to recognize symptoms of oncoming anxiety/agitation and were more involved with their care on the unit.
A Quality Improvement project was instituted on an adult acute psychiatric inpatient unit to evaluate the feasibility of music as an alternative to PRN medication for agitation. Every patient has an equal chance to ask for the music before PRN administration. The music was advertised as a coping skill option on admission.

We evaluated: 1) PRN psychiatric medication administration 3 month prior to the project and 3 months during the project; (2) patient's feedback on music helpfulness and staff feedback on music implementation and helpfulness; (3) length of stay on the unit 3 month before the music project and 3 month during the project; (4) restraints, seclusions, assaults, and property damage usage before and after the music.

\section{Results}

Diagnostic categories of patients:

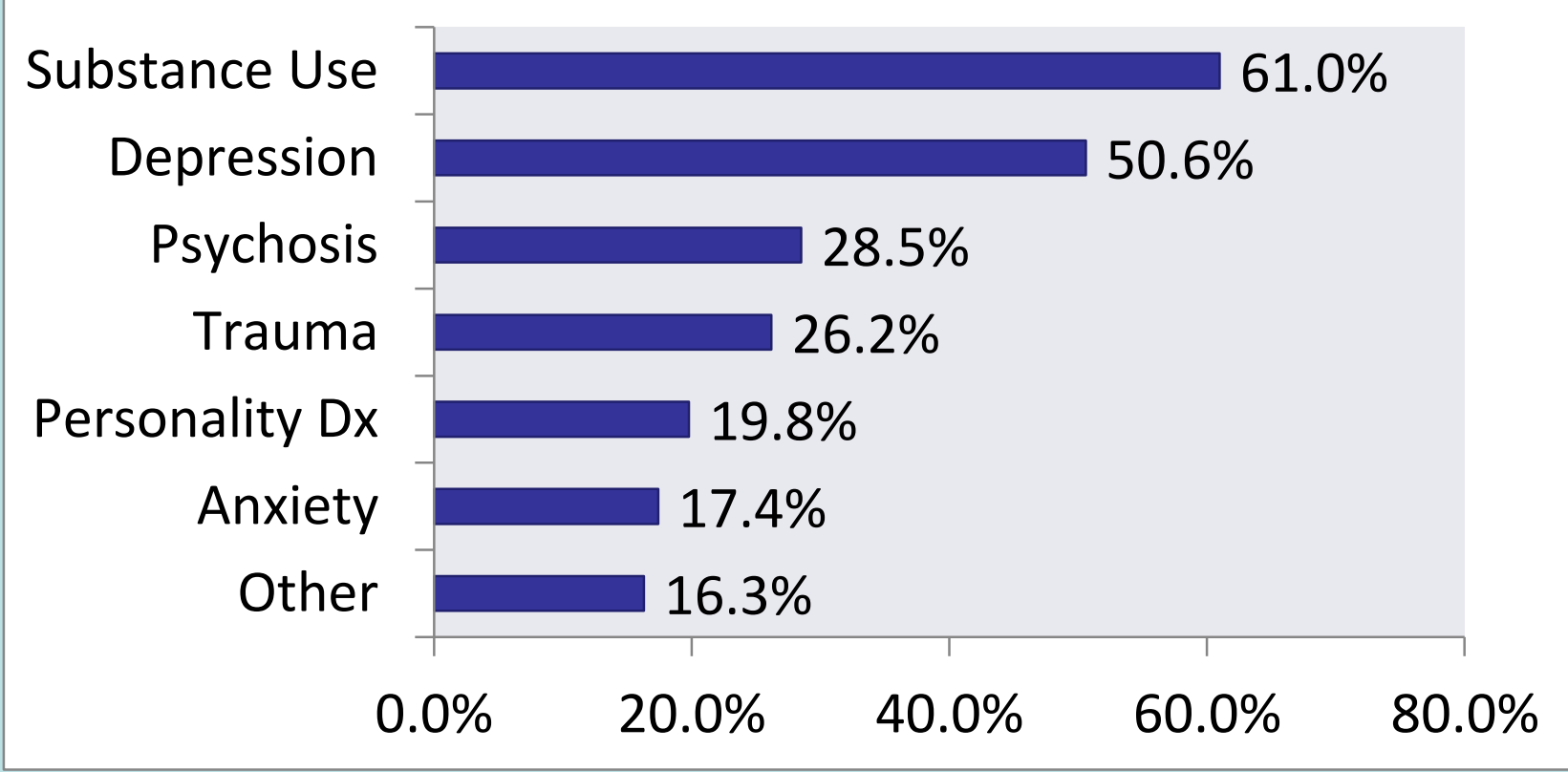

- 172 individuals, age from 18-82 yo, average = $41 \mathrm{y}$, participated in this project. The majority of patients were diagnosed with either a depressive, schizophrenia-spectrum or trauma/stressor-related illness, often with substance-use comorbidity.

- The average daily census on the unit was 17 patients. Comparing the 3 months prior to the 3 months after the PRN music intervention, the average length of stay decreased 2.29 days ( 10.49 to 8.20 days).

- Haloperidol decreased from monthly average of 15 to 8 administrations and olanzapine decreased from an average 46 to 39 administrations.

- Seclusion incidents were decreased from 26 to 12

- Assaults decreased from 3 to 0 ; property damaged went down from 2-1

- Restraints median was 2 prior to music, and 4 during the music, indicating that there were more agitated patients during that time frame.

${ }^{1}$ Yang P, L. S. (2011, January 19). Mental health implications of music; Insight from Neuroscientific and Clinical Studies. Harvard Review of Psychiatry , 34-46.

2 Baker, JA., Bowers, L., Owiti, JA. (2009). Ward features associated with high rates of medication refusal by patients: A large multi-centred survey. Gen Hosp Psychiatry, 31, 80-9. 3 Thapa, PB., Palmer, SL., Owen, RR., et al. P.R.N. (2003). (As-needed) orders and exposure of psychiatric inpatients to unnecessary psychotropic medications. Psychiatry Services, 54, 1282-6. ${ }^{4}$ Gagner-Tjellesen, D., Yurkovich, E., \& Gragert, M. (2001). Use of music therapy and other ITNIs in acute care. Journal of Psychosocial Nursing and Mental Health Services, 39 (10), 26-37.

5. Scott L. Zeller, MD 2012. Best Practice Guidelines For Agitated Patients. Psychiatric Times. 